\title{
Spectroscopy of Southern Active Stars
}

\author{
O. Vilhu ${ }^{1}$, B. Gustafsson ${ }^{2}$, and F.M. Walter ${ }^{3}$ \\ ${ }^{1}$ Observatory and Astrophysics Laboratory, University of Helsinki, \\ SF-00130 Helsinki, Finland \\ ${ }^{2}$ Uppsala Astronomical Observatory, Box 515, S-75120 Uppsala, Sweden \\ ${ }^{3}$ SUNY, Earth and Space Sciences, Stony Brook, NY 11794-2100, USA
}

\begin{abstract}
H} \alpha$ and HeI D3 lines were observed in five active cool stars over their relatively short rotation periods. Three of the stars (AB Dor, Rst137B, HD82558) are in the pre main sequence phase (as deduced from their lithium abundances), while two are post main sequence FK Comae type stars (HD32918 and BD-223467) (Vilhu et al., 1991). We present the results of these observations and point out the physical similarity of the $\mathrm{H \alpha}$ and He D3 transitions (Fig.1).
\end{abstract}

\section{Observations}

We have observed the southern active cool stars HD 32918 (K1 IIIp), HD 82558 (K2 V), BD -22 3467 (G8 III), AB Dor (HD 36705, K1 IV) and Rst 137B (M35 ), each of which lies close to the chromospheric-coronal saturation limit. The fractional $\mathrm{H} \alpha$-luminosities $\mathrm{L}_{H \alpha} / \mathrm{L}_{b o l}$ of the stars were close to $10^{-4}$. No significant radial velocity variations were detected in any of the stars. Hence, they are most probably single stars or else the companion mass is very low.

The existence of a cool spot region of HD 32918 was traced (Piskunov et al., 1990 ) by the variations of the FeI 6546-line profiles within the $9.55 \mathrm{~d}$ rotation period by a surface imaging technique. The $\mathrm{H} \alpha$-emission of $\mathrm{HD} 32918$ was variable and asymmetric, comprising possibly of chromospheric and wind (or expanding chromosphere) components.

The He I 5876 (D3) absorption was remarkably strong in HD 32918, pointing to the existence of an extended and low density chromosphere. BD-22 3467 , the cool companion to the hot central star of the planetary nebula Abell 35, exhibits a photometric wave with a 0.77 day period (Jasniewich and Acker, 1988). Our observations, performed around the same time as this photometry, resolved a stronger blue-shifted $\mathrm{H} \alpha$-emission at the minimum light (periodic $\mathrm{R} / \mathrm{V}$-variations). This behaviour was opposite to that found in HD 32918. The $v \sin i$-value $(90 \mathrm{~km} / \mathrm{s})$ of $\mathrm{BD}-22^{0} 3467$ is compatible with the interpretation that the 0.77 day photometric cycle is the star's rotation period.

The $\mathrm{H} \alpha$-emission of HD 82558 was stronger than in the previous observations by Fekel et al. (1986) and variable, but with no obvious rotational modulation. The $\mathrm{H} \alpha$-emission strengths of the young pre main sequence pair AB Dor - Rst137B are compatible with those of the Pleiades stars ( $\mathrm{W}_{H \alpha}$ vs. R-I, Stauffer and Hartmann, 1986), giving additional support to the small age of the system. Rst137B has the He I 5876 triplet in strong emission ( $E W=750 \pm 80 \mathrm{~m} \AA$ ), and its photospheric lines are broad $(v \sin i$ around $50 \mathrm{~km} / \mathrm{s})$. In $\mathrm{AB}$ Dor we detected two $\mathrm{H} \alpha$-transients (the 
first in emission and the second one in absorption). The normal quiescent state of AB Dor is the filled-in $\mathrm{H} \alpha$ (no emission nor absorption).

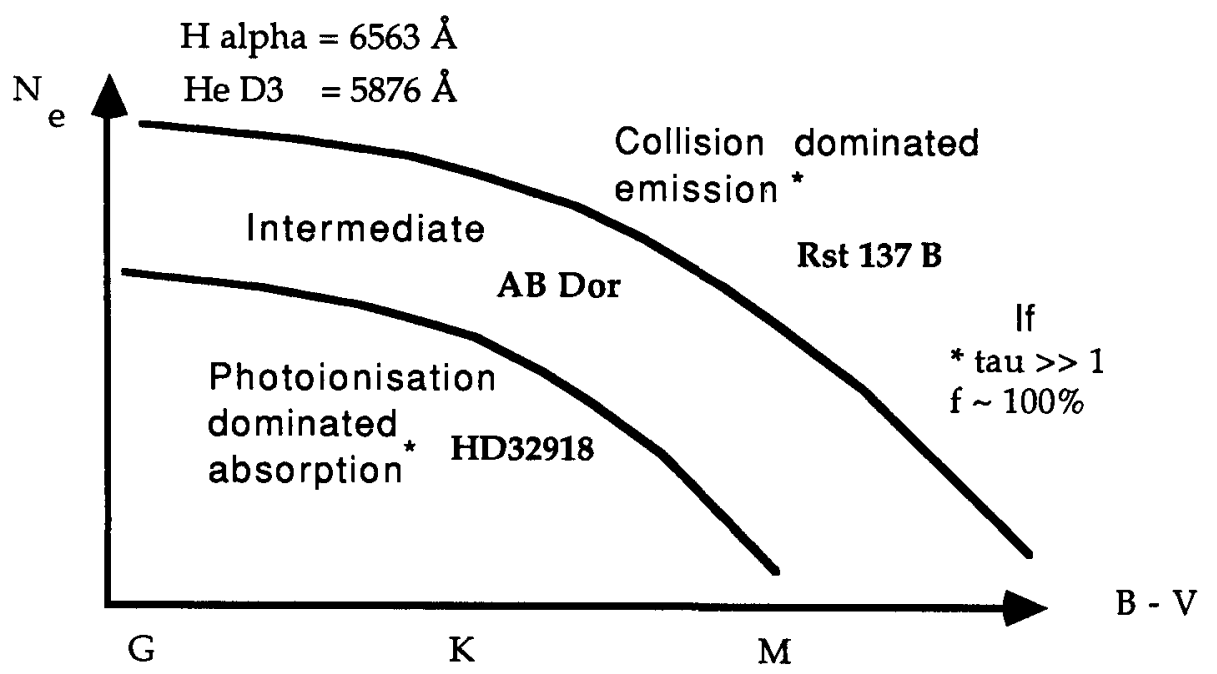

Fig. 1. A schematic representation of the $\mathrm{H} \alpha$ and He D3 lines in the spectral type chromospheric density diagram (following Giampapa and Liebert, 1986). In a low density chromosphere the lines are in absorption, while in the high density ones they appear in emission (provided that the optical depths $(\tau)$ and surface coverage filling factors (f) are large enough). The curves, separating the domains, are somewhat different for $\mathrm{H} \alpha$ and He D3.

\section{Similarities between $\mathrm{H} \alpha$ and HeI D3}

In the fastest rotators of our sample, AB Dor (0.51 d) and HD 82558 (1.66 d), we detected neither HeI D3 absorption nor emission. The physics of the $\mathrm{H} \alpha$ and HeD3 transitions seem to be related. In active and low density chromospheres (with large optical thickness and filling factor) the lines appear in absorption (photo-ionization dominated plasma), while in very high density chromospheres (Rst137B) they are in emission (collisionally dominated plasma). Intermediate cases show neither absorption nor emission (filled-in level, AB Dor as a typical example, see Fig. 1).

\section{References}

Fekel F.C., Bopp B.W., Africano J.L., Goodrich B.D., Palmer L.H., Quigley L.H., Simon T.: 1986, Astron. J. 92, 1150

Giampapa M.S., Liebert J.: 1986, Astrophys. J. 305, 784

Jasniewich G., Acker A.: 1988, Astron. Astrophys. 189, L7

Piskunov N.E., Tuominen I., Vilhu O.: 1990, Astron. Astrophys. 230, 363

Stauffer J.R., Hartmann L.: 1986, Astrophys. J. Suppl. 61, 531

Vilhu O., Gustafsson B., Walter F. 1991, Astron. Astrophys., in press 